
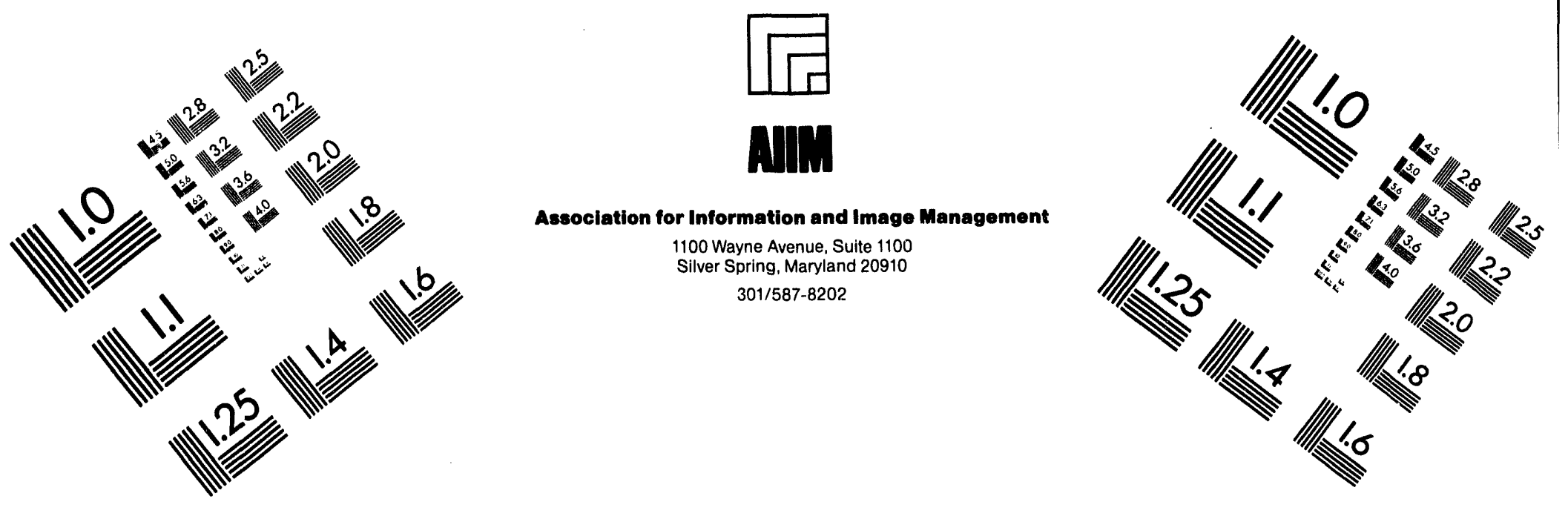

Centimeter

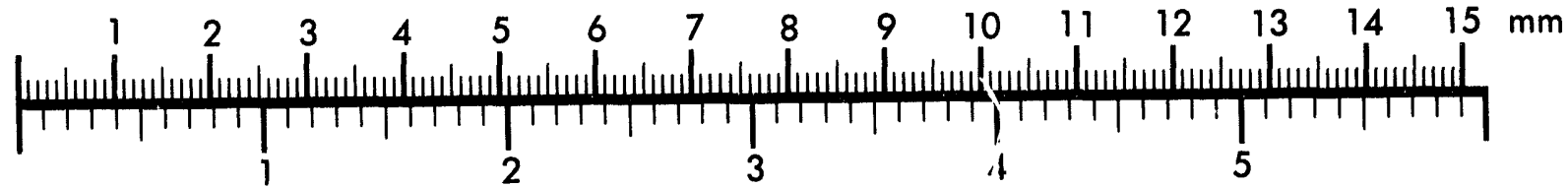
Inches
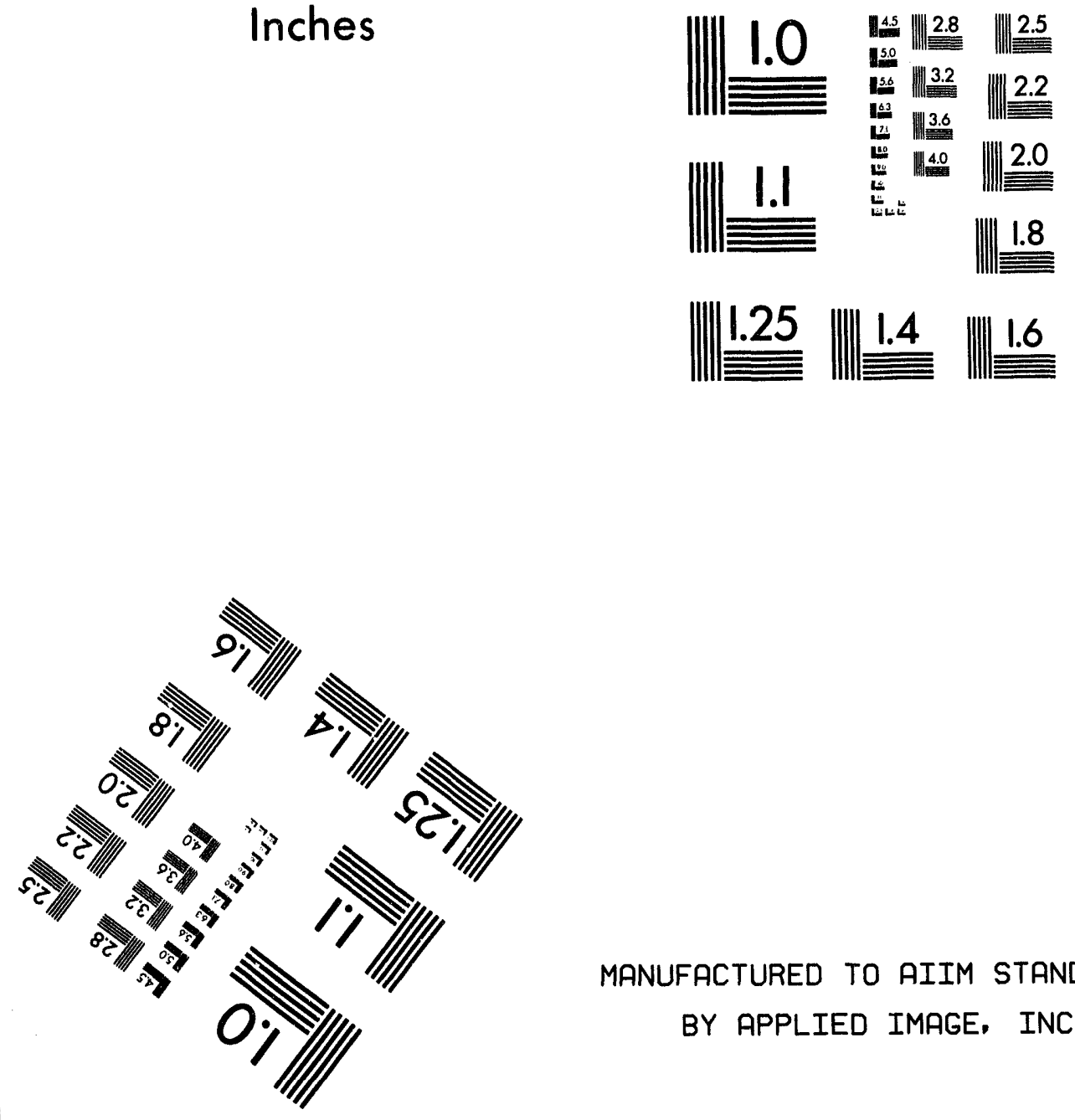

MANUFACTURED TO AIIM STANDARDS

BY APPLIED IMAGE, INC.

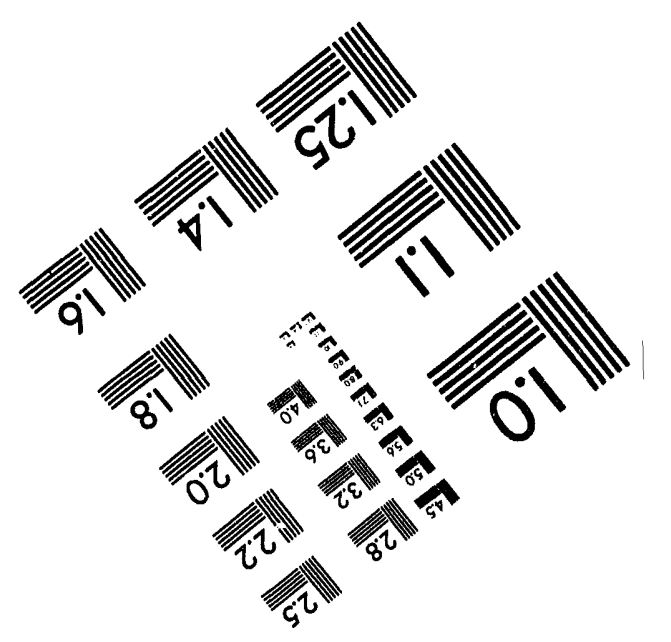



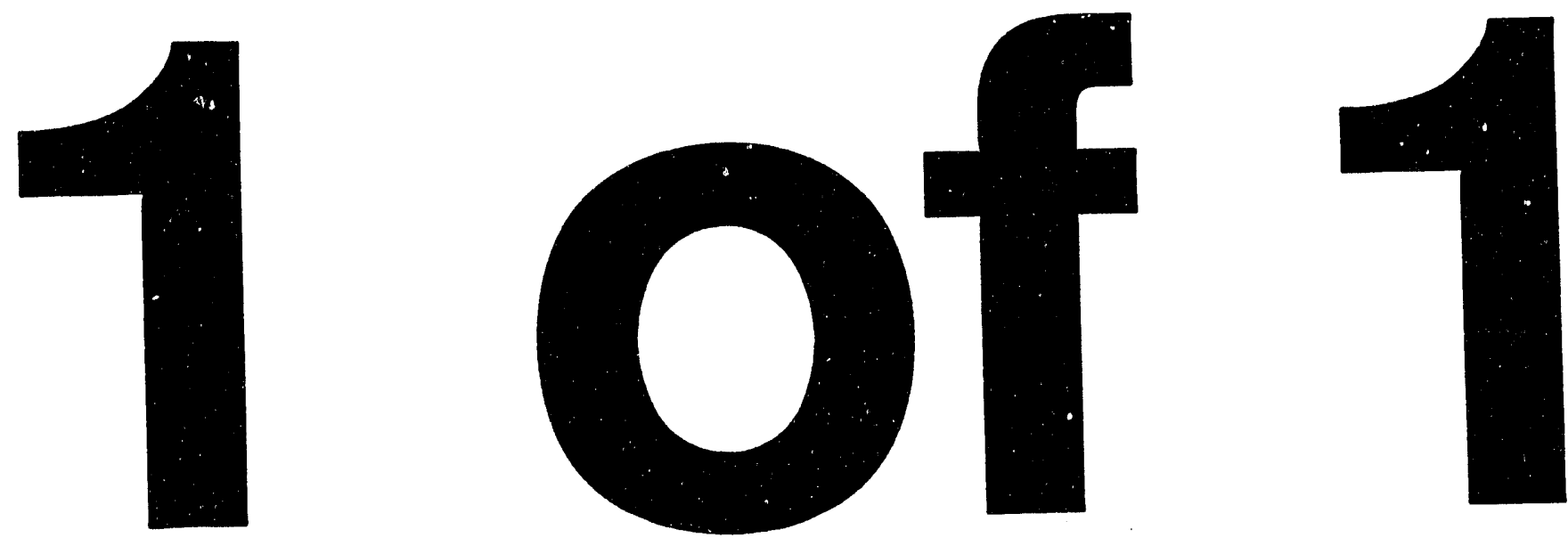
PNL-9986

UC-606

\title{
Results from the Preliminary Conveyor Evaluation of the High-Energy Beta Scintillation Sensor at the Fernald Soil Decontamination Pilot Plant
}

\author{
A. J. Schilk \\ M. A. Knopf
}

July 1994

Prepared for the U.S. Department of Energy under Contract DE-AC06-76RL0 1830

This work is funded by the Office of Technology Development, within the Department of Energy's Office of Environmental Management, under the Characterization, Monitoring, and Sensors Integrated Program.

Pacific Northwest Laboratory

Richland, Washington 99352

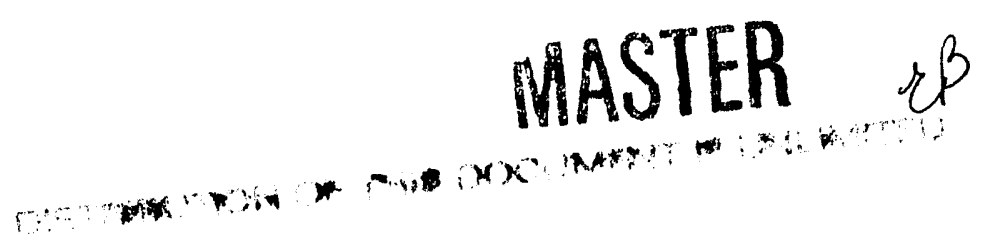




\section{Summary}

Pacific Northwest Lajoratory (PNL) tested a high-energy beta scintillation sensor to evaluate its efficacy in continually characterizing washed soils moving on a conveyor belt at Fernald's Soil Decontamination Pilot Plant (SDPP). This sensor was originally developed at PNL for monitoring uranium-contaminated soils in the field. It has a multi-layer design that enables it to discriminate against lower-energy beta particles from natural sources. It can also distinguish, to some extent, gamma rays and cosmic-induced species. The sensor detects uranium activity indirectly, based on the assumption that secular equilibrium exists between the targeted radionuclide $(234 \mathrm{mPa})$ and its parent (238U).

Several 1-h background counts were made at the SDPP each day of the evaluation period. The average background count rate was found to be comparable to that observed under previous laboratory conditions. Some elevated count rates were observed, but these were infrequent and were relatively insignificant. The sensor was calibrated both at PNL and the SDPP. Although the SDPP calibration was restricted somewhat because of conveyor-belt dimensions, the PNL data were corroborated in that the intercepts were essentially equivalent, and the regression slopes differed by a constant factor that is a direct function of the actual surface areas of the standards.

Static runs were performed on soils from SDPP test runs \#1 and \#17 to determine the extent of any residual activity following the decontamination process. Soils were found to contain $158 \pm 30$ $\mathrm{pCi} / \mathrm{g}$ and $114 \pm 25 \mathrm{pCi} / \mathrm{g}$ of $234 \mathrm{Th}-234 \mathrm{mPa}$ (238U?), respectively. But the soils had considerable moisture, and concern existed that this moisture would act as an additional beta attenuator and thereby give results that were erroneously low. Therefore, a soil sample was baked overnight, and the hard-baked clay that resulted was ground into small fragments and counted. This sample was counted for $30 \mathrm{~min}$ as before, and the total activity was determined to be $313 \pm 44 \mathrm{pCi} / \mathrm{g}$ of ${ }^{234} \mathrm{Th}$ $234 \mathrm{mPa}$ (238U?). Based on the test \#1 results, a dry-to-wet activity ratio of 1.98 has been established. Hence, if the moisture content of test \#17 was equivalent to that of test \#1, the actual $234 \mathrm{Th}-234 \mathrm{mPa}$ (238U?) activity level is expected to be approximately $226 \mathrm{pCi} / \mathrm{g}$.

For a more realistic operational scenario, the sensor was evaluated in a moving-belt configuration. Various belt speeds were used to establish dynamic calibrations as a function of relative speed. The $35-\mathrm{pCi} / \mathrm{g}$ results were compromised due to a voltage discrepancy, although a correction factor based on the static tests was incorporated, thereby permitting a three-point calibration plot for each run. A dynamic sensor evaluation reveals that the sensor response is reasonably consistent at multiple belt speeds, as well as between static and dynamic conditions.

Although the PNL beta-sensor output may not be a direct indication of the total uranium activity in some washed soils, only minor modifications to the data-reduction procedure are necessary to allow the conversion of these results to uranium activity levels. Incorporating such a sensor into the process stream would enhance the rapid and efficient decontamination of target soils. 


\section{Contents}

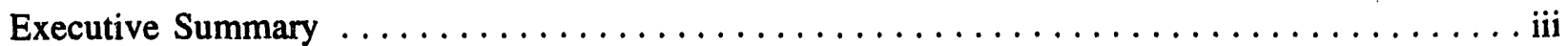

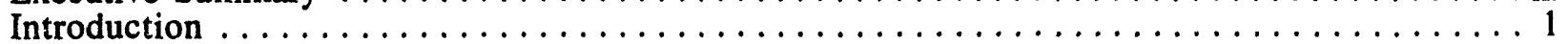

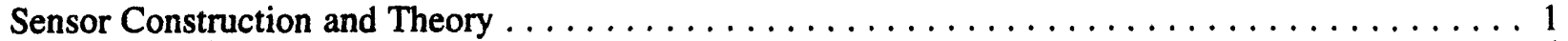

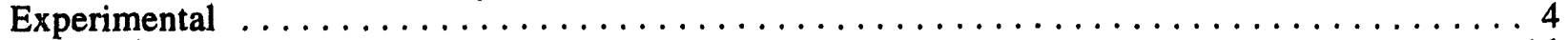

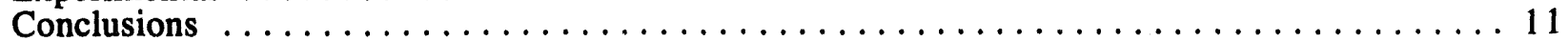

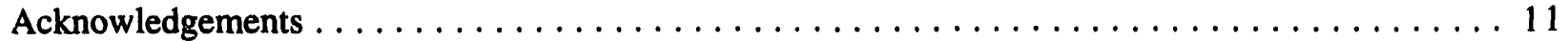

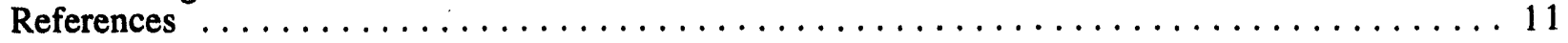

\section{Figures}

Fiqure 1. Schematic representation of the current sensor design highlighting potential interactions. Double lines denote particle tracks that lead directly to excitations/ionizations and consequent scintillations. Any triple-coincidence events that occur contemporaneously with scintillations in the overlying layer are assumed to be due to cosmic events and are therefore rejected. Triple coincidences caused by energetic Compton electrons are quite rare (due to the low-Z material of the sensor), but their likelihood is further reduced by decreasing the mass in the first (lowermost) layer.

Figure 2. A plot of the projected penetration depths of various charged species in the plastic scintillant (courtesy of BICRON Corporation, Newbury, Ohio). The polynomial describing the electron/beta behavior in this material is as follows: $\log ($ range in $\mathrm{mm})=0.0419(\log E)^{3}-0.172(\log E)^{2}+1.20(\log E)+0.572$

Fiqure 3. Typical example of a background count distribution obtained at the Fernald SDPP (histogram and cumulative plot).

Fiqure 4. Uranium calibration plots from data obtained at PNL (left) and Fernald's SDPP (right). Results from the two calibrations are equivalent following a correction for sample dimensions (i.e., PNL standards measured $30 \mathrm{~cm}$ by $60 \mathrm{~cm}$ and SDPP standards measured $20 \mathrm{~cm}$ by $60 \mathrm{~cm})$.

Fiqure 5. SDPP dynamic calibration plot-15-fpm belt speed, 15 second sample traverse time.

Fiqure 6. SDPP dynamic calibration plot-5-fpm belt speed, 44 second sample traverse time.

Figure 7. SDPP dynamic calibration plot-1-fpm belt speed, 306 to 365 second sample traverse times.

\section{Table}

Table 1. Summary of data results from SDPP dynamic sample runs. The symbol $\left(^{*}\right)$ indicates samples that were dried before evaluation. 


\section{Results from the Preliminary Conveyor Evaluation of the High Energy Beta Scintillation Sensor at the Fernald Soil Decontamination Pilot Plant}

\section{Introduction}

Pacific Northwest Laboratory (PNL)(a) has been tasked with demonstrating and evaluating the efficacy of a high-energy beta scintillation sensor for the continuous-mode characterization of washed soils from Fernald's Soil Decontamination Pilot Plant (SDPP)(b). The soil-washing process involves a series of steps that include physical attrition of the material as well as chemical stripping to remove uranium contamination and to generate an effluent that is radiologically clean and biologically viable. The need for an efficient detection methodology to continuously monitor this conveyor-belt-transported effluent, and alarm an operator if such material contains uranium activity in excess of established cutoff limits, is critical.

\section{Sensor Construction and Theory}

The sensor used for this demonstration was originally designed and constructed at PNL for use in field monitoring of uranium-contaminated soils (Schilk et al., 1993; Schilk et al., 1994) and was not modified in any fashion for this demonstration. This detector employs a vertically stacked configuration (Figure 1 ) that allows discrimination between a) unattenuated betas from $234 \mathrm{mPa}$ (a decay daughter of $238 \mathrm{U}$ ) that, when traveling at their most probable energies $(\approx 0.8 \mathrm{MeV})$, will penetrate approximately $3 \mathrm{~mm}$ of solid plastic scintillant (Figure 2), and b) lower-energy beta particles arising typically from natural sources, viz., $40 \mathrm{~K}, 232 \mathrm{Th}$ (plus daughters), and $238 \mathrm{U}$ (plus daughters). The most probable beta energies from these latter radionuclides and their progeny rarely exceed $0.3 \mathrm{MeV}$ (or their associated abundances are relatively insignificant compared with a)\}; therefore, the likely penetration depth of an unattenuated, normally incident "background" beta will generally be less than 1 to $2 \mathrm{~mm}$ (Figure 2 ) and thus easily distinguishable from a).

The current multi-layer design, with an active region measuring $30 \mathrm{~cm}$ by $60 \mathrm{~cm}$, also affords some discrimination against gamma rays and cosmic-induced species (protons, muons, etc.) by observing and partially canceling the contributions from these ubiquitous phenomena, thereby enhancing the overall sensor signal-to-noise ratio (refer to Figure 1). Cosmic influences are minimized by incorporating an overlying anti-coincidence ribbon that detects energetic charged particles originating above the sensor and rejects their contribution. A 6-mm-thick acrylic absorber separates this ribbon from the lower layers and minimizes the possibility that any soil-derived charged particles will trigger the anti-coincidence circuitry. Gamma-ray-induced triple coincidences are reduced by placing a thinner scintillating-fiber layer on the bottom of the sensor to decrease the probability of generating a Compton electron in this layer. By decreasing the number of gammarelated events in the lowermost ribbon, the total gamma triple coincidences (i.e., due to the concurrent generation of Compton electrons in all three layers by one or more incident photons or the production of a particularly energetic electron in the first layer that subsequently traverses the second and third layers) are cut back proportionately. In addition, significant improvements to the electronic components and circuitry allowed a very narrow intralayer coincidence window to be established,

a Pacific Northwest Laboratory is operated for the U.S. Department of Energy by Battelle Memorial Institute under Contract DE-AC06-76RLO 1830.

b Funding for this project has been provided by the Office of Technology Development's Characterization, Monitoring, and Sensor Technology Integrated Program under the direction of Dr. Caroline Purdy. 


\section{Cosmic-Ray Charged Emission}

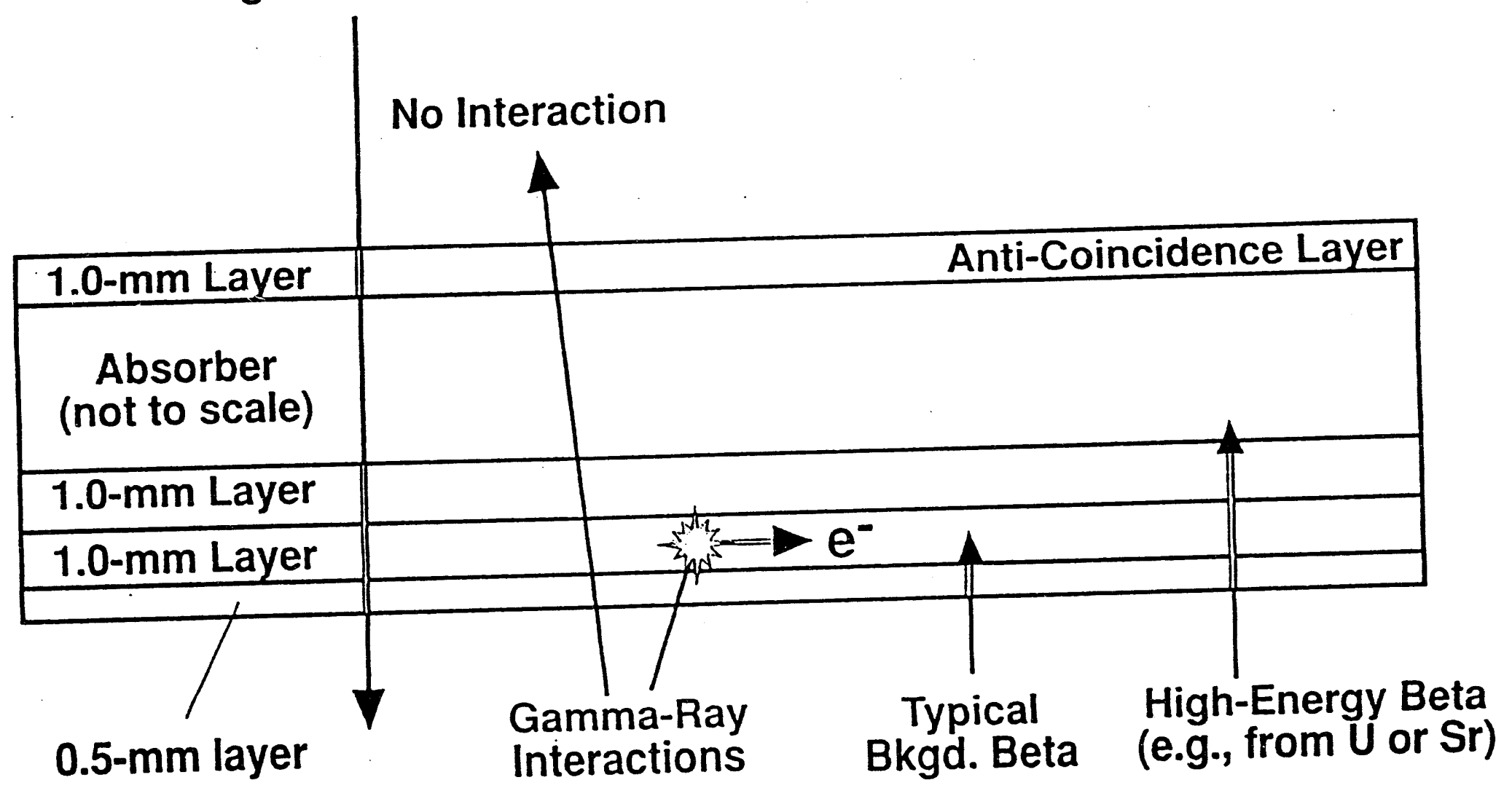

Figure 1. Schematic representation of the current sensor design highlighting potential interactions. Double lines denote Farticle tracks that lead directly to excitationsfionizations and consequent scintillations. Any triple-coincidence events that particle tracks that lead dic events and are occur cone rejected. Triple coincidences caused by energetic Compton electrons are quite rare (due to the low- $\mathrm{Z}$ material therefore rejected. Triple coincidences caused by end by decreasing the mass in the first (lowermost) layer. 


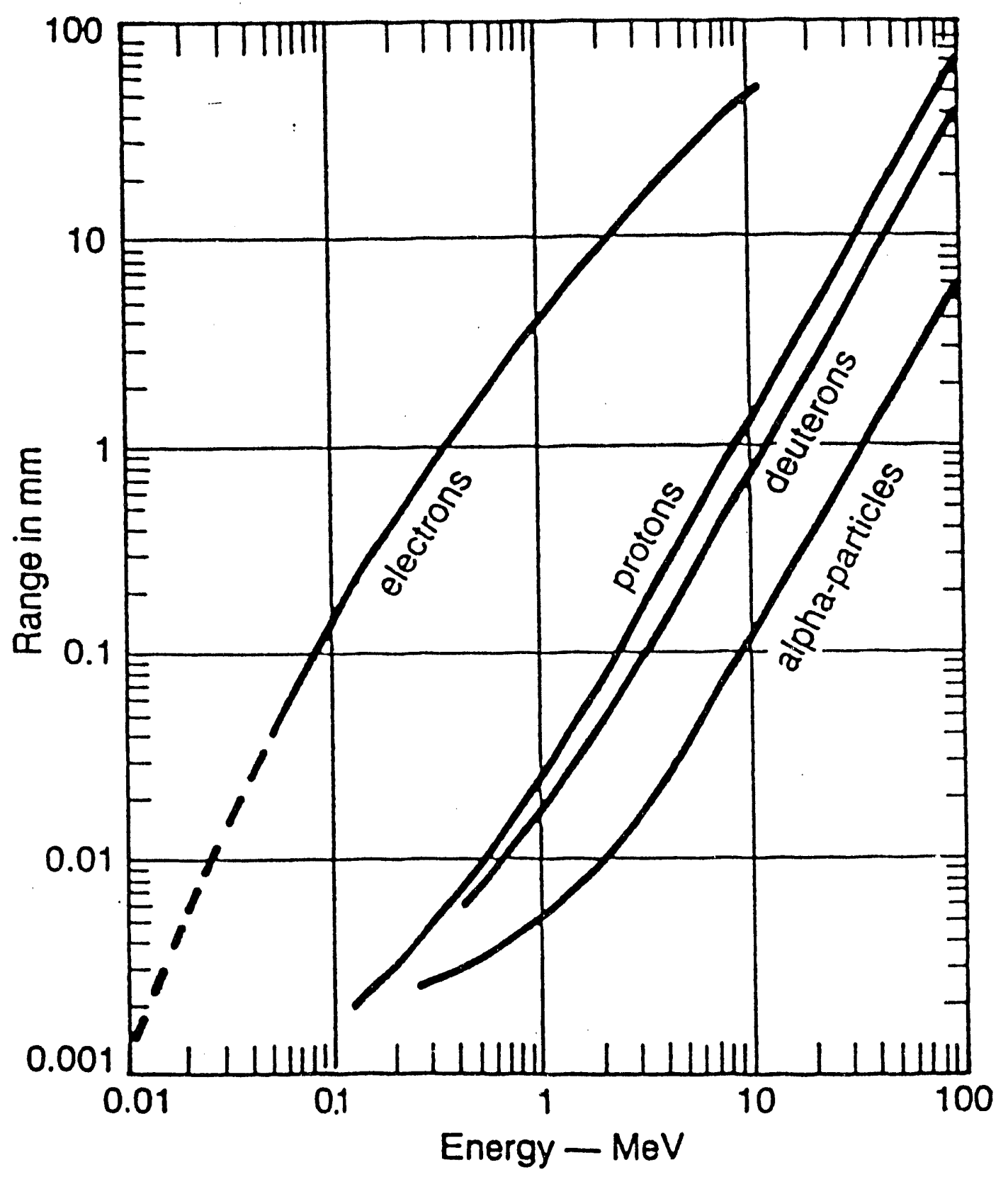

Figure 2. A plot of the projected penetration depths of various charged species in the plastic scintillant (courtesy of BICRON Corporation, Newbury, Ohio). The polynomial describing the electron/beta behavior in this material is as follows:

$\log ($ range in $\mathrm{mm})=0.0419(\log \mathrm{E})^{3}-0.172(\log )^{2}+1.20(\log \mathrm{E})+0.572$ 
thereby decreasing the background by mini:nizing the total number of potential "false positives" generated by coincident thermionic emissions from the scintillation-sensing photomultiplier tubes.

Strictly speaking, this sensor detects uranium activity indirectly, based on the assumption that secular equilibrium exists between the targeted radionuclide $(234 \mathrm{mPa})$ and its parent $(238 \mathrm{U})$. An abbreviated decay scheme is shown below:

$238 \mathrm{U}(4.47 \mathrm{E} 9 \mathrm{a})-->234 \mathrm{Th}(24.1 \mathrm{~d}) \rightarrow->234 \mathrm{mPa}(1.17 \mathrm{~m}) \ldots$... $->206 \mathrm{~Pb}$ (stable)

Indeed, this condition of equilibrium would not occur for 5 or 6 months following any separation of uranium from protactinium (or uranium from thorium) due to the presence of the relatively long-lived intermediary, ${ }^{234} \mathrm{Th}$ (note that ${ }^{234} \mathrm{Th}$ and $234 \mathrm{mPa}$ activity levels would equilibrate within $10 \mathrm{~min}$ ). With regard to contaminated soils associated with existing DOE facilities, the assumption of secular equilibrium is valid in most, if not all, cases, and the detected activity of the daughter is essentially equivalent to that of the parent isotope. For new or recently fractionated material (as is the case at Fernald's SDPP), however, one must be cognizant of potential disequilibria when using this methodology. Measuring uranium indirectly does not preclude using the highenergy beta sensor effectively for such a scenario, but necessitates understanding the 238U-234Th decontamination ratios thoroughly (i.e., the degree of uranium and thorium stripping as a function of the washing process). After these ratios have been accurately determined via an appropriate method (note that these parameters would be constant for a continuous-mode process with consistent resident times and reagent concentrations), they need only be incorporated into the sensor's data-reduction program to compensate for any uranium-protactinium (or uranium-thorium) disequilibria. Alternatively, if the ${ }^{234} \mathrm{Th}$ concentration in the soil is entirely unaffected by the decontamination process while a fraction of the uranium is removed, then it would not be possible to convert the betasensor response to $238 \mathrm{U}$ content.

One must also be cognizant of the temporal relationship between the U-(Th?-)removal stage and the monitoring stage. Normally, the latter would immediately follow the former in a continuousmode process, but if a significant delay occurs between soil stripping and effluent counting (e.g., on the order of weeks, as with the current evaluation), additional corrections will be necessary to compensate for the decay of remnant 24.1-d 234Th. As in the case of the potential U-Th disequilibrium discussed above, this phenomenon can be readily compensated for in the datareduction process.

\section{Experimental}

A number of background counts were initiated at the SDPP each day to determine the stability of the high-energy beta sensor. This involved acquiring $1-h$ counts at various times during the evaluation process while the sensor was maintained in the standard configuration (i.e., 1 to $2 \mathrm{~cm}$ above the sample surface and centered on the conveyor belt width). The average background count rate within the SDPP facility was found to be comparable to that observed under previous laboratory conditions, with a net triple-coincidence rate of $6.92+1.39$ counts per second (cps). Individual background counts exhibited near-normal distributions (e.g., Figure 3), although elevated count rates were observed in each case. It is uncertain at this point if such events were true occurrences or periodic electronic anomalies. In either case, these were infrequent enough to be relatively insignificant.

Three medium-grained sand samples were spiked with a solution of $238 \mathrm{UO}_{2}\left(\mathrm{NO}_{3}\right)_{2}$ to levels of 5 , 15 , and $35 \mathrm{pCi}$ of $238 \mathrm{U}$ (and $234 \mathrm{Th}, 234 \mathrm{mPa}$ ) per gram to facilitate the sensor calibration. These sources were first characterized at PNL in a $30-\mathrm{cm}$ by $60-\mathrm{cm}$ by $1-\mathrm{cm}$ geometry (to match the sensor's active area and approximate an infinite soil depth for 2.3-MeV betas), and the results are summarized in Figure 4 (left). The non-zero intercept is a manifestation of the primordial uranium activity present in the control sand. An additional calibration was subsequently performed at the SDPP, although in this 


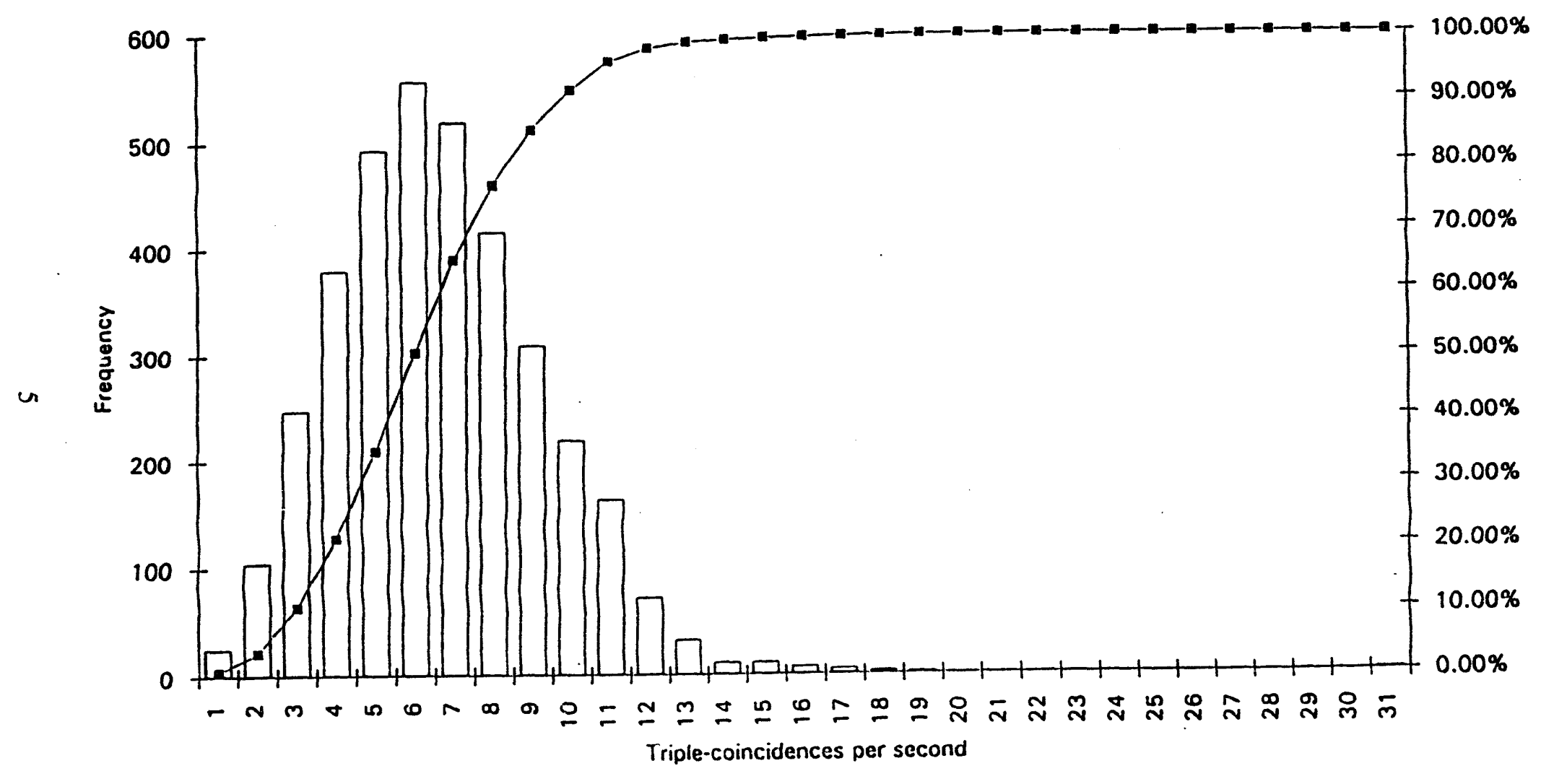

Figure 3. Typical example of a background count distribution obtained at the Fernald SDPP (histogram and cumulative plot). 

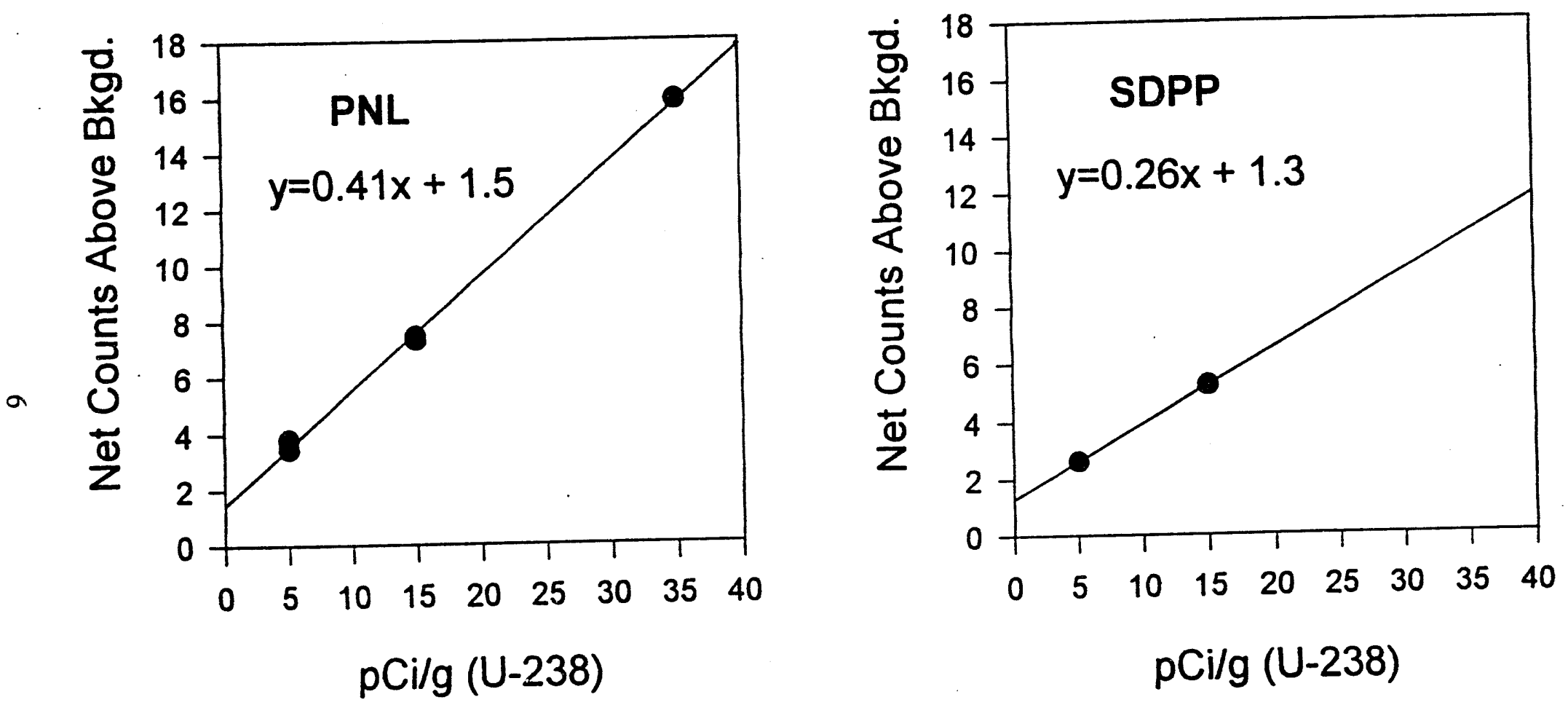

Figure 4. Uranium calibration plots from data obtained at PNL (left) and Fernald's SDPP (right). Results from the two calibrations are equivalent following a correction for sample dimensions (i.e., PNL standards measured $30 \mathrm{~cm}$ by $60 \mathrm{~cm}$ and SDPP standards measured $20 \mathrm{~cm}$ by $60 \mathrm{~cm}$ ). 
case the width of the soil standard was restricted to $20 \mathrm{~cm}$ due to the conveyor-belt dimensions. The $35-\mathrm{pCi} / \mathrm{g}$ measurement was compromised as a result of improper voltage settings and thus only two data points were obtained. Nonetheless, the SDPP calibration (Figure 4; right) corroborates the PNL data in that the intercepts are essentially equivalent and the regression slopes differ by a constant factor that is a direct function of the actual surface areas of the standards. Specifically, the slope of the SDPP calibration plot is $63 \%$ of that associated with the PNL plot (i.e., 0.26 vs. 0.41 ), while the active soil area for the SDPP runs was roughly two-thirds, or $67 \%$, of the PNL standards.

Static runs were then performed on soils from SDPP test runs \#1 and \#17 to determine the extent of any residual activity following the decontamination process. Soil aliquots were found to be quite damp and rather spongy in texture, the latter perhaps due to the addition of an organic material for inducing flocculation. All samples were arranged in the same geometry as the previous standards and were counted for $30 \mathrm{~min}$ each. Based on the aforementioned calibration, test \#1 and \#17 soils were found to contain $158 \pm 30 \mathrm{pCi} / \mathrm{g}$ and $114 \pm 25 \mathrm{pCi} / \mathrm{g}$ of $234 \mathrm{Th}-234 \mathrm{mPa}$ (238U?), respectively.

Some concern existed with regard to the moisture content of these soils and the potential effects on the sensor response due to the presence of interstitial water. This excessive moisture (approx. 50 wt.\% water) was expected to act as an additional beta attenuator and thereby to give results that were erroneously low. Hence, an aliquot from test \#1 was collected and heated overnight in a suitable oven to eliminate all adsorbed water in the sample. The dried sample had the consistency of baked clay (again, probably due to the binding properties of the flocculent material) and, to facilitate counting, the sample was subjected to a crushing process that created irregular fragments ranging in size from fine dust to approximately $6 \mathrm{~mm}$. This sample was counted for $30 \mathrm{~min}$ as before, and the total activity was determined to be $313 \pm 44 \mathrm{pCi} / \mathrm{g}$ of $234 \mathrm{Th}-234 \mathrm{mPa}$ (238U?). Based on the test \#1 results, a dry-towet activity ratio of 1.98 has been established. Therefore, if the moisture content of test \#17 was equivalent to that of test \#1, the actual $234 \mathrm{Th}-234 \mathrm{mPa}(238 \mathrm{U}$ ?) activity level is expected to be approximately $226 \mathrm{pCi} / \mathrm{g}$.

The sensor was also evaluated in a moving-belt configuration, which is a more realistic operational scenario with respect to the projected continuous-mode decontamination facility at Fernald. In this investigation, the same sand standards (i.e., 5, 15, and $35 \mathrm{pCi} / \mathrm{g}$ ) were passed $1-2 \mathrm{~cm}$ below the sensor at various belt speeds to establish dynamic calibrations as a function of relative speed. As mentioned above, the $35-\mathrm{pCi} / \mathrm{g}$ results were compromised due to a voltage discrepancy, although a correction factor based on the static tests was incorporated, thereby permitting a threepoint calibration plot for each run. Specifically, the $35-\mathrm{pCi} / \mathrm{g}$ data point from the SDPP calibration was found to be approximately $24 \%$ low with respect to the remaining points and, therefore, all $35-$ $\mathrm{pCi} / \mathrm{g}$ results from the dynamic runs were multiplied by a factor of 1.24 .

Figures 5-7 summarize the results for the three individual belt speeds of approximately 1, 5, and $15 \mathrm{feet} /$ minute (fpm). The calibrations were based on the net cumulative counts obtained during each traverse of a $20-\mathrm{cm}$ by $60-\mathrm{cm}$ by $1-\mathrm{cm}$ soil standard, from the time at which the standard's leading edge reached the sensitive area of the sensor to the point when the standard's trailing edge left this region. Damp soil samples from test runs \#1, \#4, \#16, and \#17 were monitored under equivalent conditions, and net counts were determined as above (note: dried aliquots of test runs \#l and \#16 were similarly evaluated for comparative purposes). During the nominal 15 -fpm and 5-fpm runs, each soil sample spent a total of $15 \mathrm{sec}$ and $44 \mathrm{sec}$, respectively, under the sensor's active area. Although the conveyor-belt motor controller indicated the same speed values for each of the 1-fpm runs, the actual sample traverse times ranged from 305 to $365 \mathrm{sec}$ (nonetheless, this "roving" parameter did not appear to induce significant errors into these runs). 


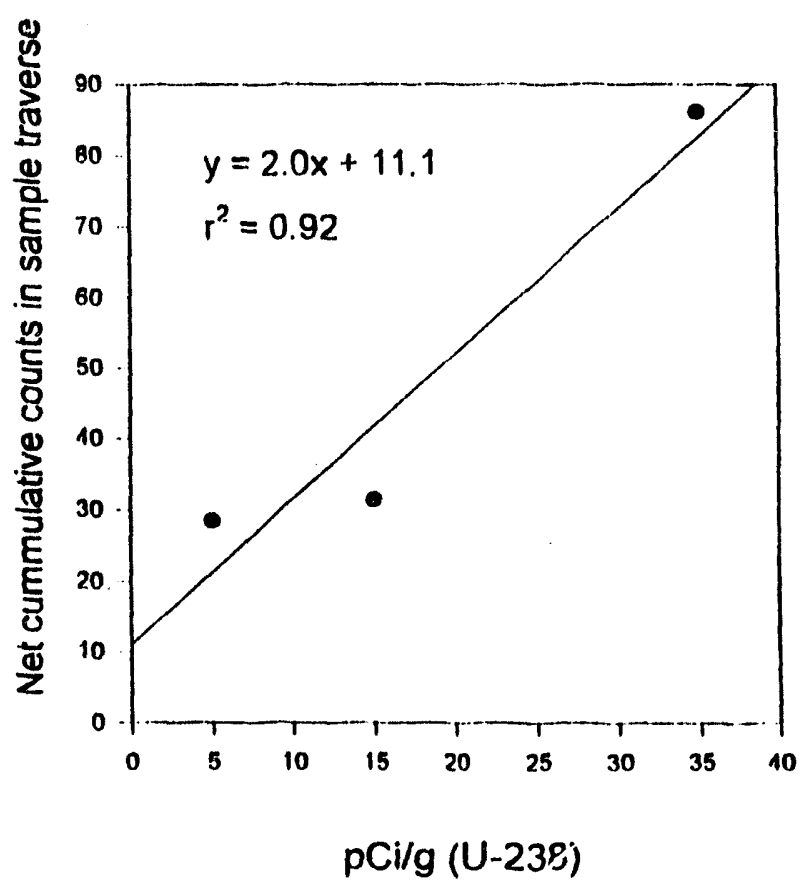

Figure 5. SDPP dynamic calibration plot -- 15-fpm belt speed, 15 second sample traverse time.

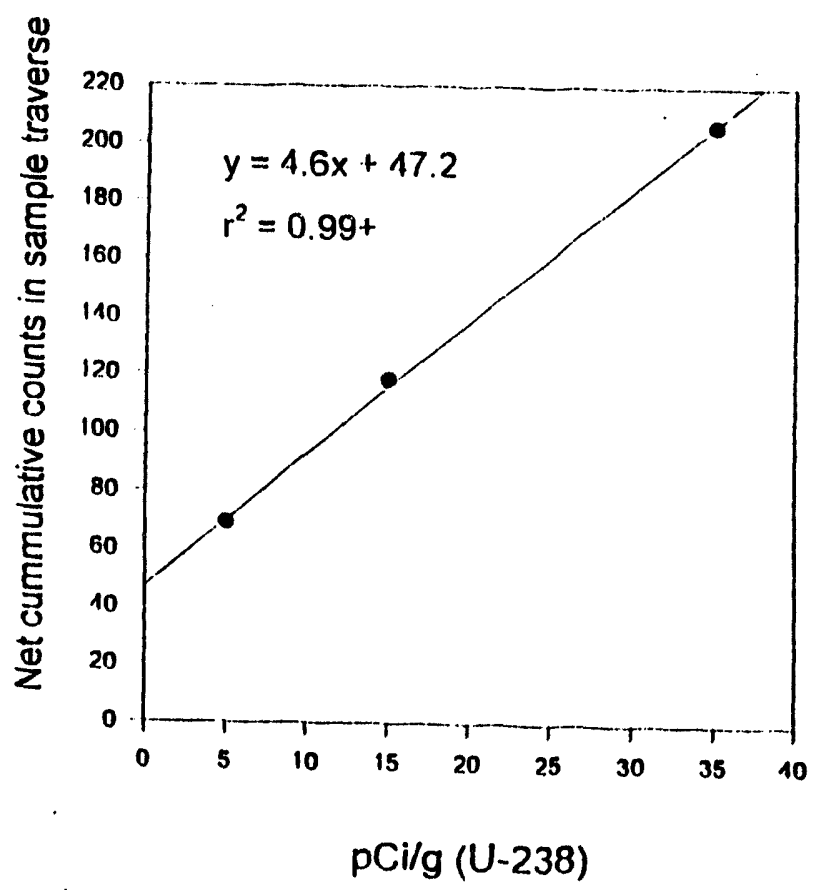

Figure 6. SDPP dynamic calibration plot -- 5-fpm belt speed, 44 second sample traverse time. 


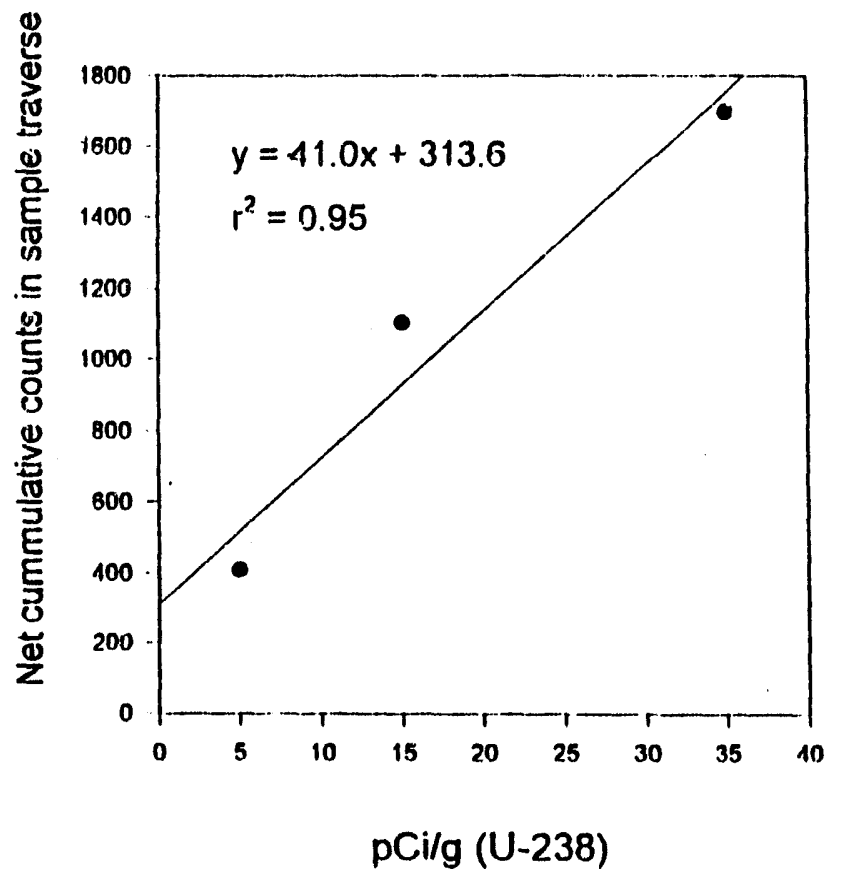

Figure 7. SDPP dynamic calibration plot -- 1-fpm belt speed, 306 to 365 second sample traverse times.

Table 1 is a compilation of the results of the dynamic sensor evaluation. It is apparent from these results that the sensor response is reasonably consistent at multiple belt speeds, as well as between static and dynamic conditions (as evidenced by the data from test runs \#1 and \#17). Standard deviations for the dynamic measurements were determined by assuming pure Poisson statistics (i.e., $1 \sigma$ errors are equal to the square root of the total counts), which led to relative errors that were somewhat less than those derived empirically from the complementary static measurements. Errors associated with the static runs included contributions from other sources of error in addition to those related simply to the stochastic process of radioactive decay (e.g., electronic fluctuations in the sensor). Hence, true $1 \sigma$ errors for the dynamic results are likely to be somewhat higher than those indicated in Table 1, further enhancing data overlap. 
Table 1. Summary of data results from SDPP dynamic sample runs. The symbol $\left(^{*}\right)$ indicates samples that were dried before evaluation.

\begin{tabular}{|c|c|c|}
\hline & Thorium-234 (pCi/g) & 1 - sigma s.d. (pCi $/ g)$ \\
\hline \multicolumn{3}{|l|}{ Test \#1 } \\
\hline Static run & 158 & $30(19 \%)$ \\
\hline 15-fpm run & 149 & $12(8 \%)$ \\
\hline 5-fpm run $A$ & 198 & $14(7 \%)$ \\
\hline 5-fpm run $B$ & 174 & $13(8 \%)$ \\
\hline 1 -fpm run & 149 & $12(8 \%)$ \\
\hline Static run* & 313 & $45(14 \%)$ \\
\hline 15-fpm run* & 309 & $18(6 \%)$ \\
\hline 5-fpm run $A^{*}$ & 401 & $20(5 \%)$ \\
\hline 5-fpm run $B^{*}$ & 376 & $19(5 \%)$ \\
\hline 1-fpm run* & 309 & $18(6 \%)$ \\
\hline \multicolumn{3}{|l|}{ Test \#4 } \\
\hline 15-fpm run & 59 & $8(13 \%)$ \\
\hline 5-fpm run $A$ & 79 & $9(11 \%)$ \\
\hline 5-fpm run B & 75 & $9(12 \%)$ \\
\hline 1-fpm run & 69 & $8(12 \%)$ \\
\hline \multicolumn{3}{|l|}{ Test\#16 } \\
\hline 15 -fpm run & 123 & $11(9 \%)$ \\
\hline 5-fpm run $A$ & 179 & $13(7 \%)$ \\
\hline 5-fpm run B & 182 & $13(7 \%)$ \\
\hline 1-fpm run & 142 & $12(8 \%)$ \\
\hline 15-fpm run* & 221 & $15(7 \%)$ \\
\hline 5-fpm run $A^{*}$ & 248 & $16(6 \%)$ \\
\hline 5-fpm run $B^{*}$ & 259 & $16(6 \%)$ \\
\hline 1-fpm run* & 202 & $14(7 \%)$ \\
\hline \multicolumn{3}{|l|}{ Test \#17 } \\
\hline Static run & 114 & $25(22 \%)$ \\
\hline 15-fpm run & 100 & $10(10 \%)$ \\
\hline 5-fpm run $A$ & 152 & $12(8 \%)$ \\
\hline 5-fpm run B & 149 & $12(8 \%)$ \\
\hline 1-fpm run & 121 & $11(9 \%)$ \\
\hline
\end{tabular}




\section{Conclusions}

At this point, and before any radiological assays to determine the extent of potential 238U-234Th $(234 \mathrm{mPa})$ disequilibria created by the soil-washing process, it would be imprudent to claim that the dry-sample results obtained from the SDPP evaluation (Table 1) are equivalent to effluent uranium concentrations. These results, however, are certainly indicative of the 234Th content in the samples and, as mentioned previously, can be converted to $238 \mathrm{U}$ activity levels if 1) specific conditions exist (e.g., $U$ and Th must both be affected to some degree by the physical/chemical stripping process) and 2) information regarding the U-Th decontamination ratio (normally, a constant parameter) is available.

Currently, PNL investigators are performing radiochemical and spectrometric analyses of soil samples from each of the aforementioned test runs to establish $238 \mathrm{U}$ and $234 \mathrm{Th}$ concentrations in each. Efforts are underway to obtain fresh (i.e., unwashed, influent) soil from the Fernald site to evaluate pre-processing concentrations and to determine uranium and thorium decontamination ratios. These data, combined with decay corrections to compensate for the delay period between sample processing and monitoring, should allow the accurate characterization of uranium content in each aliquot.

In summary, although the PNL beta-sensor output may not be a direct indication of the total uranium activity in some washed soils (unlike with earlier field evaluations at Fernald), only minor modifications to the data-reduction procedure are necessary to allow the conversion of these results to uranium activity levels. Incorporating such a sensor into the process stream (following a suitable calibration that addresses the issues described above) would enhance the rapid and efficient decontamination of target soils.

\section{Acknowledgments}

The authors would like to express their appreciation to the following FERMCO personnel without whose assistance this demonstration could not have been completed: Rochelle Chernikoff, Jane Phend, Matt Arnett, and John Goines. Thanks are also due to Kim Nuhfer (FERMCO; ID Coordinator) for her support and guidance. We are indebted to Caroline Purdy of the the Office of Technology Development, within the Department of Energy's Office of Environmental Management, under the Characterization, Monitoring, and Sensors Integrated Program.

\section{References}

Schilk, A. J., R. W. Perkins, K. H. Abel, R. L. Brodzinski, D. P. Brown, R. C. Thompson, and M. A. Knopf. 1993. "Quantitative In Situ Uranium Contaminant Mapping using High-Resolution Gamma-Ray Spectrometry and Beta Scintillation Counting," PNL-SA-23141, Pacific Northwest Laboratory, Richland, Washington. Presented at the ER' 93 Environmental Remediation Conference, Oct. 24-28, Augusta, Georgia.

Schilk, A. J., K. H. Abel, and R. W. Perkins. 1994. Characterization of Uranium Contamination in Surface and Subsurface Soils, PNL-SA-23787, Pacific Northwest Laboratory, Richland, Washington. Accepted for publication in the J. Environ. Radioact. 


\section{Distribution}

No. of

Copies

\section{Oftsite}

12 DOE/Office of Scientific and Technical Information

C. B. Purdy

U.S. Department of Energy 19901 Germantown Road

Germantown, MD 20874

10 K. R. Nuhfer

FERMCO

P.O. Box 398704

MS-8 1

Cincinnati, OH 45239-8704
No. of

Copies

\section{Onsite}

DOE Richland Operations Office

D. E. Trader

17 Pacific Northwest Laboratory

A. J. Schilk (6)

M. A. Knopf

D. E. Robertson

S. C. Slate (3)

Publishing Coordination

Technical Report Files (5)

Dist. 1 

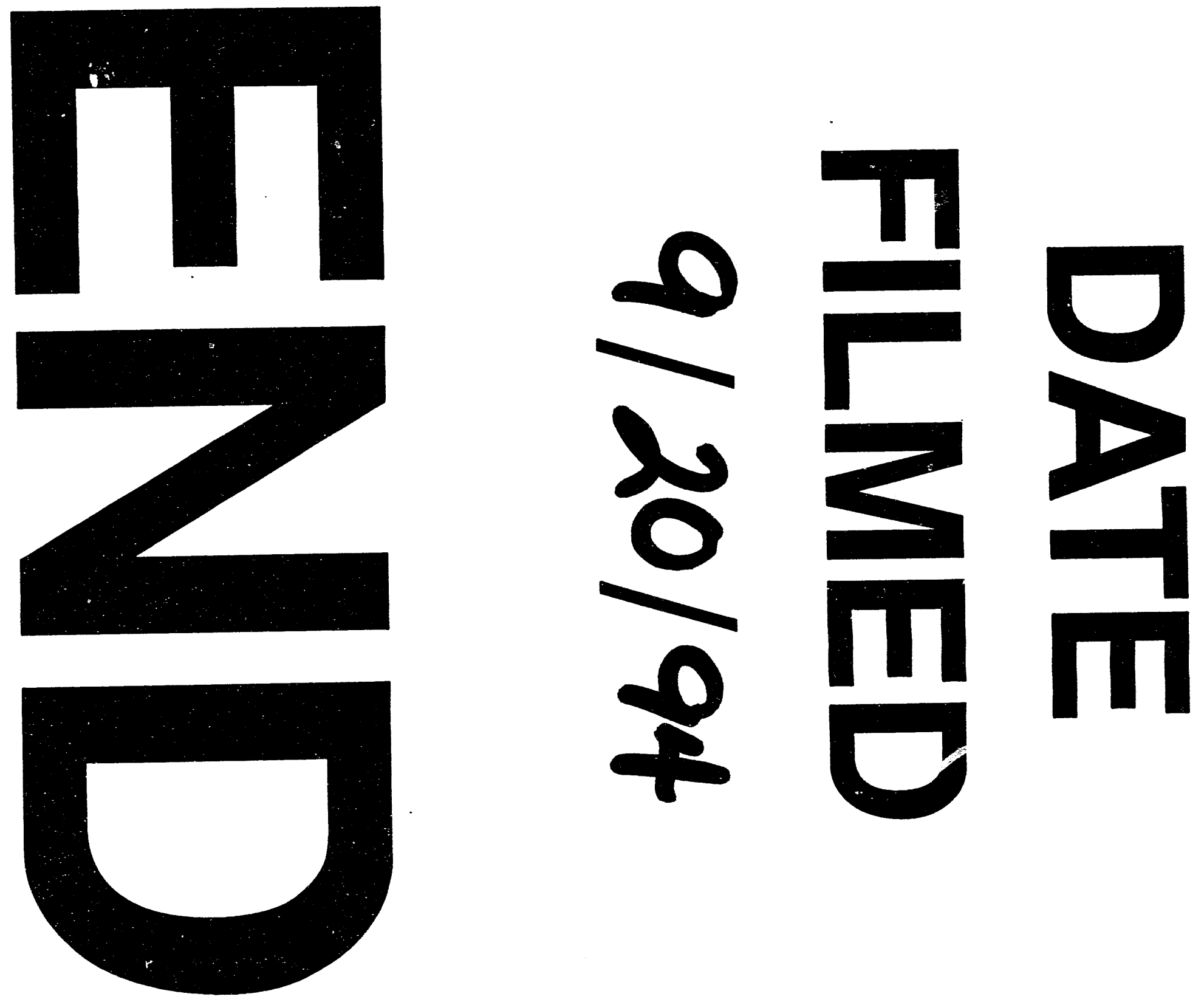
